\title{
The Precise Description of Pumping Rod string and Tubing Movement and Computer Implementation
}

\author{
Yang MengShi, a ${ }^{1,}$ Xie Wei, ${ }^{1, b}$ Jin XueFeng ${ }^{1, ~ a, ~}$, Li Yun ${ }^{1, ~ a ~}$, Liu TingTing, a , Zeng

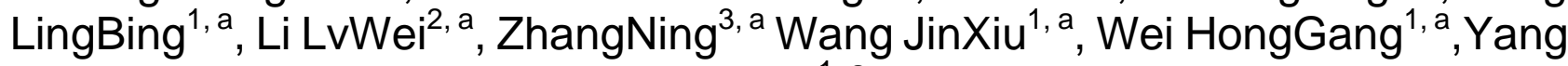 \\ Song ${ }^{1, a}$ \\ ${ }^{1}$ Petroleum Production Engineering Research Institute of PetrochinaHuabei Oilfield Company, \\ Renqiu city, Hebei province, China \\ ${ }^{2}$ Exploration and Development Research institute of PetrochinaHuabei Oilfield Company Renqiu \\ city, Hebei province, China \\ ${ }^{3}$ The Fourth Exploit Factory of PetrchinaHuabeiOifield Company Langfang city,Hebei province, \\ China

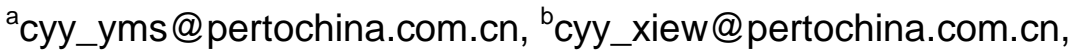

Keywords:Rod string mechanics, Finite element,Computer implementation..

Abstract.Discussedseveral common mathematical model on well force research field, and build a rod string motion law simulation system base on the model of finite element, which can directly display the movement rule of the pumping unit, the well trajectory, the axial movement of the rod string, the rule of the radial movement of the rod string, the contact pressure of the rod string and so on. Andcalculated ten production wells, verify the reliability of the simulation system.

\section{Preface}

After many years research on therod string, the understanding of the rod string motion law is more and more deeply, achieved many academic achievements, yet in terms of production, the calculation results given by the model are mostly not intuitive; In terms of scientific research, oil production engineering research lacks of experimental method, lack of test wells, or the test fee is expensive, and the procedure is cumbersome, and sometimes disturb the daily production. Therefore, From the scientific research point of view is also very much hope that there is a simple efficient and inexpensive test method, so we need a simulation software that can show the real well working conditions and guide the field production, and be a substitute for the field test.

The core of a simulation software is the mathematical model, the rationality of the model directly determine the accuracy of the simulation result. currently the rod string model mainly focus on axial displacement description, but in the actual production, the rod string dose not only move in one dimension, to show the rod string work state accurately, also needs radial motion law of rod string.

\section{Current Major Models}

Axial movement model of rod string.The sucker rod string moving reciprocally in the tubing, transfer kinetic energy, this kind of simulation model is comparatively mature, there are a many axial vibration mathematical model, usually use the wave equation, a mature method, to study the axial 
vibration of sucker rod string, but the effects of the transverse vibration and torsional vibration on the rod string are rarely considered, is not accurate enough.

Rod string buckling model and contact pressure analysis.On up stroke, the rod string is tensioned cause of gravity, on down stroke, the rod string is generally divided into three status, stability, sinusoidal buckling, and helical buckling. Due to the Counterforce from pump end, the internal stress of sucker rod string at the lower end increased, when the internal stress reaches a certain point, sucker rod string at the lower end start to bent, with the increase of the counterforce from the pump end, the rod string and tubing contact, the rod string is under sinusoidal buckling state or even helical buck-ling state ${ }^{[1]}$. Due to the influence of borehole trajectory, the stiffness of the tubing and the weight of the rod string, the buckling load of the rod string are difficult to be accurately determined. This makes it difficult to determine the configuration of the rod string, and can only be used as an auxiliary model.

Three dimension finite element model of sucker rod string.At present, there have been certain researches on the three dimension Finite element mod-el of sucker rod string in China. such as GU Yong's Research and Application of Mechanics model of Sucker Rod string by Finite Element Method ${ }^{[2]}$, Yao Chundong's ${ }^{[3]} A$ Finite Element Simulation Model of the Rod-tubing Contact State in Directional Wells ${ }^{[3]}$. These model are based on the finite element method, it analyzed the rod string, and can reflect the axial motion law accurately.

The basic idea of the finite element model of rod string is to first divided the rod string into a number of discrete "beam element", then focus on one beam element, establish cell balance equation based on the principle of virtual work, through coordinate conversion assembled the overall stiffness matrix and load vector, then establish the overall balance equation of rod string ${ }^{[4,5,6]}$. Compared to other types of models, this model has a very good description on the axial, radial displacement of the rod string, is suitable as a core model for the program.

To use finite element model to solve the problem usually need to use ABAQUS, ANSYS or other professional finite element computing software, although this kind of software is powerful, has wide range of applications, but to solve the problem the preprocessing stage needs rich experience, the solving process usually requires a lot of computation, and one simulation often take hours or even days, while in the actual production of-ten requires a plurality of wells, the application of the finite element method is limited by the large amount of computation, and the high precision has exceeded the actual work demand. In order to enable it to play a role in daily work, In the programming process, we cut down the sampling accuracy, achieved a balance between accuracy and computing speed, so it won't affect the accuracy, and within a relatively short period of time to complete the simulation..

\section{Model Establishment}

Discrete the rod string into many beam elements, both ends of the beam elements has displacement vector $\{\bar{\delta}\}^{e}$, and force vector $\{\bar{F}\}^{e}$, the elastic potential energy of each beam elements is:

$$
\prod_{P}=\int_{L} \frac{1}{2}\{\varepsilon\}^{\mathrm{T}}[\mathrm{D}]\{\varepsilon\} \mathrm{dl}-\int_{\mathrm{L}}\{\mu\}^{\mathrm{T}}\{\mathrm{q}\} \mathrm{dl}-\left(\{\bar{\delta}\}^{\mathrm{e}}\right)^{\mathrm{T}}\{\overline{\mathrm{F}}\}^{\mathrm{e}}
$$


Where $\mathrm{L}=$ Beam elements length; $\{\varepsilon\} 、\{q\}=$ Strain vector and load vector distribution; $[D]=$ Elasticity matrix; $\mu$ element displacement vector;

Obtained by the principle of minimum potential energy:

$$
\{\overline{\mathrm{F}}\}^{\mathrm{e}}=\{\overline{\mathrm{K}}\}^{\mathrm{e}}\{\bar{\delta}\}^{\mathrm{e}}
$$

Stiffness matrix is:

$$
\{\overline{\mathrm{K}}\}^{\mathrm{e}}=\int_{\mathrm{L}}\{\mathrm{B}\}^{\mathrm{T}}\{\mathrm{D}\}\{\mathrm{B}\} \mathrm{dx}
$$

Combine the element stiffness matrix, establish the overall structure stiffness matrix, in the same train of thought, obtaining node load of the whole structure, and get the overall balance equation:

$$
\{\overline{\mathrm{F}}\}=[\mathrm{K}]\{\delta\}
$$

After introducing structural boundary conditions, singularity of the overall element stiffness matrix are eliminated, the overall balance equations can be solved,obtain the node displacement $\{\delta\}$ 。

In order to describe the contact state of the rod string and tubing, add slidable spring element on space beam element node, each node attached two spring elements, spring element only constraint radial displacement of the nodes, has no intersect with the tubing, and has no constraint to the axial displacement and angular displacement of the node (Fig.1),combine the beam element and spring element,This nonlinear mechanics system can be solved.

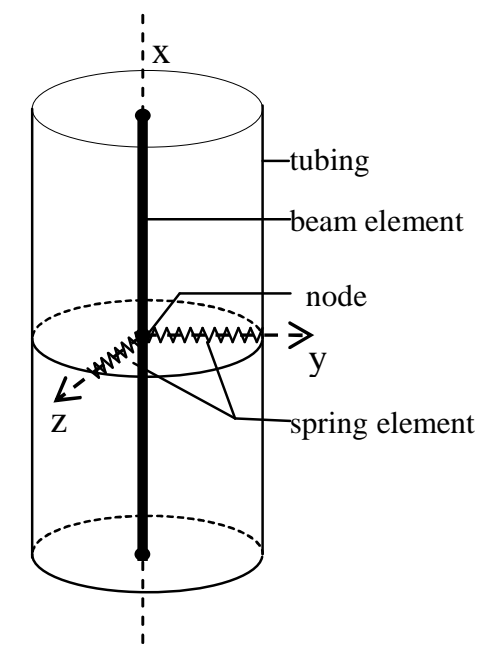

Figure 1. Spring element distribution on the node

Let there are $n$ nodes after discrete, if the spring element stiffness coefficient on number $i$ nod in $\mathrm{y}$-axis and z-axis direction is $k_{y i}$ and $k_{z i}$, and nodal displacement is $\bar{v}_{i}$ and $\bar{w}_{i}$, then the reaction forces in $\mathrm{y}$-axis and $\mathrm{z}$-axis direction are:

$$
\begin{aligned}
& \mathrm{F}_{\mathrm{iy}}=-\mathrm{k}_{\mathrm{yi}} \overline{\mathrm{v}}_{\mathrm{i}} \\
& \mathrm{F}_{\mathrm{iz}}=-\mathrm{k}_{\mathrm{zi}} \overline{\mathrm{w}}_{\mathrm{i}}
\end{aligned}
$$


Let $k_{p, p}$ and $k_{q, q}$ are the diagonal elements of the overall stiffness matrix in row $p$ and row $q$, and $p=6(i-1)+1, q=6(i-1)+3,(i=1,2, \ldots n)$, their corresponding external loads are $F_{p}$ and $F_{q}$, Thereby obtaining:

$$
\left\{\mathrm{k}_{\mathrm{p}, 1}, \mathrm{k}_{\mathrm{p}, 2}, \ldots, \mathrm{k}_{\mathrm{p}, \mathrm{p}}, \ldots, \mathrm{k}_{\mathrm{p}, 6 \mathrm{n}}\right\}\{\delta\}=\mathrm{F}_{\mathrm{p}}-\mathrm{k}_{\mathrm{yi}} \overline{\mathrm{v}}_{\mathrm{i}}
$$

Where $\left\{k_{p, 1}, k_{p, 2}, \ldots, k_{p, p}, \ldots, k_{p, 6 n}\right\}$ are the elements of row $p$ of the overall stiffness matrix, $\{\delta\}$ is overall nodal displacement matrix.

Transpose $-k_{y i} \bar{v}_{l}$ to the left side of equation:

$$
\left\{\mathrm{k}_{\mathrm{p}, 1}, \mathrm{k}_{\mathrm{p}, 2}, \ldots, \mathrm{k}_{\mathrm{p}, \mathrm{p}}+\mathrm{k}_{\mathrm{yi}}, \ldots, \mathrm{k}_{\mathrm{p}, 6 \mathrm{n}}\right\}\{\delta\}=\mathrm{F}_{\mathrm{p}}
$$

In the same way:

$$
\left\{\mathrm{k}_{\mathrm{q}, 1}, \mathrm{k}_{\mathrm{q}, 2}, \ldots, \mathrm{k}_{\mathrm{q}, \mathrm{q}}+\mathrm{k}_{\mathrm{zi}}, \ldots, \mathrm{k}_{\mathrm{q}, 6 \mathrm{n}}\right\}\{\delta\}=\mathrm{F}_{\mathrm{q}}
$$

As can be seen from the above equation,consider the influence of the spring element,Just add the stiffness coefficient of spring element to corresponding main diagonal elements of the overall stiffness matrix, the structural balance equation become:

$$
\left[\begin{array}{ccccccc}
\mathrm{k}_{1,1} & \mathrm{k}_{1,2} & \cdots & \cdots & \cdots & \cdots & \mathrm{k}_{1,6 \mathrm{n}} \\
\mathrm{k}_{2,1} & \mathrm{k}_{2,1} & \cdots & \cdots & \cdots & \cdots & \mathrm{k}_{2,6 \mathrm{n}} \\
\vdots & \vdots & \vdots & \vdots & \vdots & \vdots & \vdots \\
\mathrm{k}_{\mathrm{p}, 1} & \mathrm{k}_{\mathrm{p}, 2} & \cdots & \mathrm{k}_{\mathrm{p}, \mathrm{p}}+\mathrm{k}_{\mathrm{yi}} & \cdots & \cdots & \mathrm{k}_{\mathrm{p}, 6 \mathrm{n}} \\
\mathrm{k}_{\mathrm{q}, 1} & \mathrm{k}_{\mathrm{q}, 2} & \cdots & \cdots & \mathrm{k}_{\mathrm{q}, \mathrm{q}}+\mathrm{k}_{\mathrm{zi}} & \cdots & \mathrm{k}_{\mathrm{q}, 6 \mathrm{n}} \\
\vdots & \vdots & \vdots & \vdots & \vdots & \vdots & \vdots \\
\mathrm{k}_{6 \mathrm{n}, 1} & \mathrm{k}_{6 \mathrm{n}, 2} & \cdots & \cdots & \cdots & \cdots & \mathrm{k}_{6 \mathrm{n}, 6 \mathrm{n}}
\end{array}\right]\left[\begin{array}{c}
\delta_{1} \\
\delta_{2} \\
\vdots \\
\delta_{\mathrm{p}} \\
\delta_{\mathrm{q}} \\
\vdots \\
\delta_{6 \mathrm{n}}
\end{array}\right]=\left[\begin{array}{c}
\mathrm{F}_{1} \\
\mathrm{~F}_{2} \\
\vdots \\
\mathrm{F}_{\mathrm{p}} \\
\mathrm{F}_{\mathrm{q}} \\
\vdots \\
\mathrm{F}_{6 \mathrm{n}}
\end{array}\right]
$$

Since the contact boundary conditions of the rod string and the tubing is unknown,the overall balance equation is difficult to solve, therefore, the boundary conditions must first be processed before solving the structural balance equations.

Whether the rod stringcontacts with the tubing or not, should be determined by the discriminant 11 below:

$\sqrt{\overline{\mathrm{v}}_{\mathrm{i}}^{2}+\overline{\mathrm{w}}_{\mathrm{i}}^{2}}>\delta_{\mathrm{i}}$

$\delta_{i} \longrightarrow$ the difference between tubing internal radius and sucker rod string radius;

$\bar{v}_{i}$ — the radial displacement of the ith node on y-axis under local coordinates;

$\bar{w}_{i} \longrightarrow$ the radial displacement of the ith node on $\mathrm{z}$-axis under local coordinates;

If the radial displacement of the rod string meets the discriminamt equation (11),this problem is a nonlinear contact problem, and needs multiple judgment and iterations, Otherwise is a free boundary. 
For the free boundary, solve the structural balance equations without add the spring element, get the displacement of each node, for the node meets the discriminant, Add the stiffness coefficient $K_{i}$ to the spring element according to how much ${\sqrt{\bar{v}_{i}}{ }^{2}+\bar{w}_{i}^{2}}^{2}$ larger then $\delta_{i}$ to constraints the nodes.

Then, addedthe stiffness coefficient $K_{i}$ to the nodes, solve the structural balance equations, for the nodes still meets the discriminant, adjustment thestiffness coefficient $K_{i}$ and recalculate again, until the nodal displacement meets the iteration accuracy.

After the iteration, the rod string and tubing contact pressure $F_{i}$ can be obtained as follows:

$$
F_{i}=\sqrt{\left(k_{y i} \times \bar{v}_{i}\right)^{2}+\left(k_{z i} \times \bar{w}_{i}\right)^{2}}
$$

In the same way,the pressure angle of the contact point can be determined.

\section{Software Implementation}

Basic Architecture.The software using VB language, C/S architecture, support Windows XP and above version, the software is divided into three layers, including data layer, model layer and presentation layer.

The program has a local database, will save the basic information and simulation results automatically. The parameters for calculation can be input manually, or input by an Excel or a TXT file, and with the access permission, the program can connect external database, transfer required data from these databases directly in real-time.

Finite Element Analysis Module.The advantage of finite element method is that it can accurately calculating axial force, radial force, radial displacement, and the contact pressure at any point and time of the rod string. This is of great significance to user decision (Fig.2).

To analyze rod string with finite element method, will require to decompose the rod string motion in a cycle into several moments, by calculating the gesture of rod strings in every moment, the movement law of the rod sting in the whole cycle in obtained. But the cycle decomposition will greatly increase the operation quantity, requires a lot of computing resources, the calculation time is often up to several hours, therefore in order to re-duce computation time and improve efficiency, program has been simplified processing in the operation process ,time node is reduced, and by difference to complement simplified node. The user can select the number of computing nodes according to their needs. In ensuring the accuracy of the premise, usually choice 30 points, the computation time can be controlled in about 10 minutes, greatly improving the work efficiency of the user. 

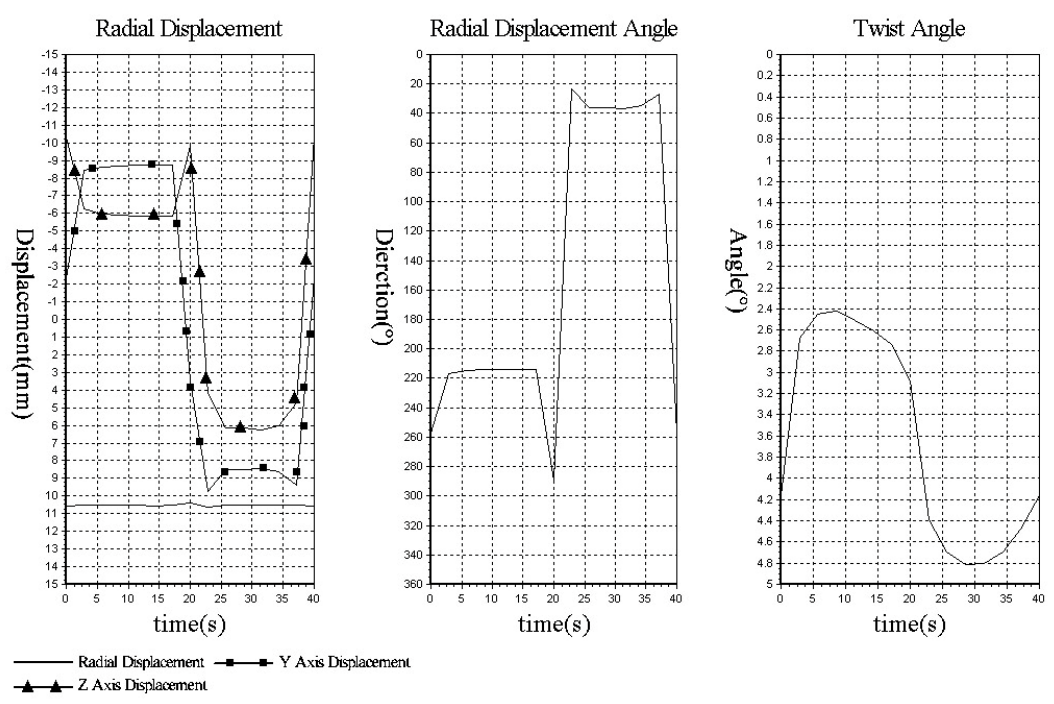

Figure 2.Nod radial displacement

\section{Practical Application}

In order to verify the accuracy of the software, use the software to simulate the actual production wells, selected 10 production wells to simulated calculations.

Table 1. Running Time

\begin{tabular}{lccccccccccc}
\hline $\begin{array}{l}\text { Will } \\
\text { number }\end{array}$ & $125 \mathrm{X}$ & $814 \mathrm{X}$ & $815 \mathrm{X}$ & $816 \mathrm{X}$ & $823 \mathrm{X}$ & $316 \mathrm{X}$ & $317 \mathrm{X}$ & $318 \mathrm{X}$ & $319 \mathrm{X}$ & $322 \mathrm{X}$ & Average \\
\hline Time(min) & 13.3 & 12.4 & 11.5 & 10.8 & 9.2 & 9.5 & 10.2 & 11.3 & 9.2 & 11.8 & 10.9
\end{tabular}

As it can be seen from Table 1, the ten wells longest operation time is 13 minutes, the shortest is 9 minutes, and it is relates to the shape and depth of the well. It takes an average of 11 minutes for a finite element simulation, and compared with finite element software ANSYS, the software greatly improving operational efficiency. At the same time, the calculation accuracy of the results can also meet the needs of practical work.

From the simulation results, selected the hole sections with larger rod tubing contact pressure, and compare with the actual pump inspection job records. Ignore the pump inspection cause by non-eccentric wear factors, only concerned operations such as rod tubing replacement and centralizer add caused by rod string fracture or tubing leak, the results in Table 2:

Table 2.comparison of simulation results and pump inspection record

\begin{tabular}{llllll}
\hline $\begin{array}{l}\text { Will } \\
\text { number }\end{array}$ & $\begin{array}{l}\text { High stress } \\
\text { position(m) }\end{array}$ & $\begin{array}{l}\text { Eccentric } \\
\text { wear } \\
\text { position(m) }\end{array}$ & Will number & $\begin{array}{l}\text { High stress } \\
\text { Position(m) }\end{array}$ & $\begin{array}{l}\text { Eccentric } \\
\text { wear } \\
\text { position(m) }\end{array}$ \\
\hline $11-25$ & $530-1000$ & $530-1100$ & $31-16 \mathrm{X}$ & $820-940$ & $815-910$ \\
$814 \mathrm{X}$ & $350-600$ & $395-580$ & $31-17 \mathrm{X}$ & $740-1000$ & $750-970$ \\
$815 \mathrm{X}$ & $100-350$ & $115-350$ & $31-18 \mathrm{X}$ & $710-1210$ & $730-1100$ \\
$816 \mathrm{X}$ & $220-360$ & $200-400$ & $31-19 \mathrm{X}$ & $500-1100$ & $500-1080$ \\
$823 \mathrm{X}$ & $540-700$ & $550-690$ & $31-22 \mathrm{X}$ & $600-750$ & $550-800$ \\
\hline
\end{tabular}

As can be seen from Table 2, the position of rod tubing replacement and centralizer add operation of actual production wells are basically consistent with the simulation results, which proved that the 
software is able to simulate the movement of the rod string in a relatively short period of time using the finite element method, it has a certain practicality.

\section{Conclusions}

Compiled the rod string fine simulation system by using finite element model, after calculation, found that the simulation results and the actual partial wear position are basically consistent, the software can provide basis for partial wear position prediction and the se-lection of the installation position of the stabilizer. The simulation results also shows that the simplify to the simulation process is reasonable, the description of rod string movement can meet the demand the accuracy requirements of engineering.

\section{References}

[1] LIU FengWu, GAO DeLi..An Analysis of Sinusoidal Buckling of Long Tublars Subject to Radial Con-straint, Engineering Mechanics ,1000-4750,2002,19(6):44 48

[2] GU Yong, Research and Application of Mechanics model of Sucker Rod string by Finite Element Meth-od,Inner Mongolia Petrochemical Industry:1006-7981,2008,13:114-117

[3] Yao Chundong, A Finite Element Simulation Model of the Rod-tubing Contact State in Directional Wells, China Petroleum Machinery:1001-4578,2010,38(11):28-32

[4] TAN MeiLan, A Set of Efficient Displacement Functions for Arbitrarily Spatial Curved Rod Elements, Engineering Mechanics :1000-4750,2004,21(3):134-137

[5] WANG XuCheng, SHAO Min, Basic principles and numerical methods of finite element meth-od,Tsinghua University Press,2003

[6] LvHeXiang, Jiang HeYang, Nonlinear finite element, Chemical Industry Press,1992 